\title{
Application of Data Fusion Technology Based on Weight Improved Particle Swarm Optimization Neural Network Algorithm in Wireless Sensor Networks
}

\author{
Xiajun Ding, Hongbo Bi, Xiaodan Jiang and Lu zhang \\ College of Electrical and Information Engineering, Quzhou University, Quzhou, Zhejiang, \\ China \\ dxj_yl@163.com
}

\begin{abstract}
With the development of sensor technology, network technology, embedded control technology and wireless communication technology, the application of wireless sensor networks (WSN) has become more and more widely. Wireless sensor networks have been named the most influential and important technology of the world in twenty-first Century. In wireless sensor networks, data fusion is an important research branch. In this paper, a data prediction model of wireless sensor network based on weight improved particle swarm optimization neural network algorithm is proposed. In view of the deficiency of the traditional BP neural network model, this paper combines with the characteristics of the data prediction model, and the BP neural network model is improved and integrated. After that, we train the neural network's sample set, and add the momentum item to correct the weight, so that the neural network can be predicted more quickly and accurately. The main idea of this paper is to predict the future data based on the historical data which are collected by sensor nodes, so as to achieve the purpose of reducing the amount of data transmission in the network and saving the energy of nodes. Finally, the experimental results show that the improved particle swarm optimization algorithm based on weight improved particle swarm optimization neural network algorithm has higher accuracy than the multiple regression method and the grey prediction method. In addition, the method can be used to effectively save energy in wireless sensor data transmission.
\end{abstract}

Keywords: wireless sensor networks; prediction; data fusion technology; BP neural network

\section{Introduction}

With the development of sensor technology, network technology, embedded control technology and wireless communication technology, the application of wireless sensor networks (WSN) has become more and more widely. Wireless sensor networks have been named the most influential and important technology of the world in twenty-first Century [1-2]. The key technology of data fusion has an important influence on the performance of wireless sensor networks. Because WSN has the advantages of self organization, rapid deployment, high fault tolerance and strong concealment and so on, it is very suitable for the battlefield target location [3], physiological data collection [4], intelligent transportation system [5], ocean detection [6] and other fields. However, the wireless sensor network still faces many technical challenges. For example, the storage capacity and processing capacity of sensor node is very limited, the network bandwidth is small, and the quality of transmission service is not high enough. Therefore, the new technology is needed to optimize the wireless sensor networks, so that it can be used in various fields. Data fusion is one of the important research fields in wireless sensor networks. The role of data fusion is mainly embodied in the aspects of saving energy, improving the 
efficiency of data collection, enhancing the accuracy of data, and obtaining comprehensive information. The emergence of data fusion makes the research focus of wireless sensor networks extended from address to data [7-9]. There are three main types of data collection in wireless sensor networks. They are timely query, cycle reporting and event reporting [10-13]. According to the characteristics of the WSN data collection, there will be a lot of redundant or wrong information about the data which are collected by the surrounding monitoring objects. If we transmit information which is not processed, it will make the node energy excessive consumption, so as to shorten the network life cycle. Therefore, we must use the data processing technology to reduce the redundancy or wrong information after the node collects the data information. In addition, the wireless sensor network can also use this technology to reduce the negative impact of node failure. The data fusion technology can reduce the node energy consumption and improve the accuracy of information, but it may also increase the network delay and robustness.

In this paper, a data prediction model of wireless sensor network based on weight improved particle swarm optimization neural network algorithm is proposed. In view of the deficiency of the traditional BP neural network model, this paper combines with the characteristics of the data prediction model, and the BP neural network model is improved and integrated. After that, we train the neural network's sample set, and add the momentum item to correct the weight, so that the neural network can be predicted more quickly and accurately. The main idea of this paper is to predict the future data based on the historical data which are collected by sensor nodes, so as to achieve the purpose of reducing the amount of data transmission in the network and saving the energy of nodes. Finally, the experimental results show that the improved particle swarm optimization algorithm based on weight improved particle swarm optimization neural network algorithm has higher accuracy than the multiple regression method and the grey prediction method. In addition, the method can be used to effectively save energy in wireless sensor data transmission. The main contents of this paper are as follows: the second part introduces the basic knowledge of wireless sensor networks. The third and four parts introduce the neural network and particle swarm optimization. The fifth part introduces the weight improved neural network algorithm of particle swarm optimization. The sixth part is the simulation experiment and the analysis of the nature. The last is the summary of the full article.

\section{Basic Knowledge of Wireless Sensor Networks}

RFID technology uses radio frequency signals to identify target objects and read the relevant information of the object, which reflects the characteristics of the object and describes the static characteristics of the object [14]. For each object in wireless sensor networks, it is necessary to consider the change of their physical state, and to record their dynamic characteristics in the environment. In this respect, WSN plays an important role in bridging the gap between the physical and virtual worlds. A large number of sensor nodes are deployed in a random or artificial way in the monitoring area, and they construct the network by self-organizing method [15]. Through the multi hop routing of nodes in the network, it transmit the data from the sensor node to the sink node. Finally, through satellite, Internet or wireless access server to arrive the terminal management node. In this way, the user can use management node to execute a series of feedback operation to WSN. Such as configuration management, task distribution, security control, etc [16-17]. 


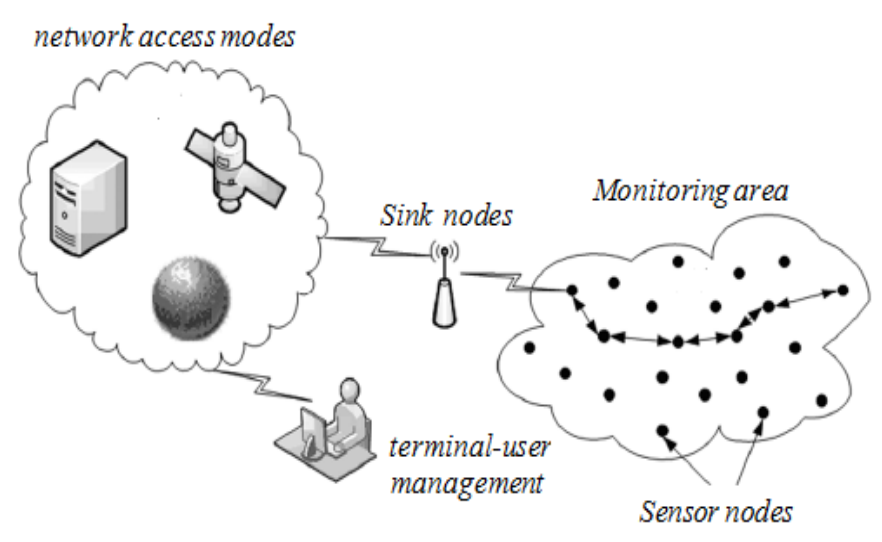

Figure 1. WSNs Architecture

\section{Neural Network Mode}

BP neural network is a multilayer feedforward neural network. It belongs to the error back propagation algorithm. It is composed of input layer, output layer and some hidden layer. Each layer has a plurality of nodes, each node represents a node of neuron with a connection between the upper and the lower nodes, and the nodes of the layer are connected with the layer by a full interconnection, and there is no association between each layer. As shown in figure 2.

The BP algorithm usually uses the sigmoid function as the excitation function. Next, we give several commonly used excitation functions.

(1) Threshold excitation function:

$$
f(x)= \begin{cases}1, & x \geq 0 \\ 0, & x \leq 0\end{cases}
$$

(2) Sigmoid excitation function:

$$
f(x)=\frac{1}{1+e^{-x}}
$$

(3) Linear excitation function:

$$
f(x)=k x
$$

(4) Hyperbolic tangent excitation function:

$$
f(x)=\tan \left(\frac{x}{T}\right)
$$

In this paper, we use the formula 2 as an excitation function of BP neural network. It satisfies the strict increasing property, it can make the output show a good balance in the linear and nonlinear. So, it can realize the input and output of any nonlinear mapping, and suitable for medium and long-term forecast. This function has the advantages of good approximation effect, fast calculation speed and high accuracy. At the same time, it's theory basis is perfect, the derivation process is rigorous, and the formula is symmetrical. It has strong nonlinear fitting ability, and it is suitable for small data processing. 


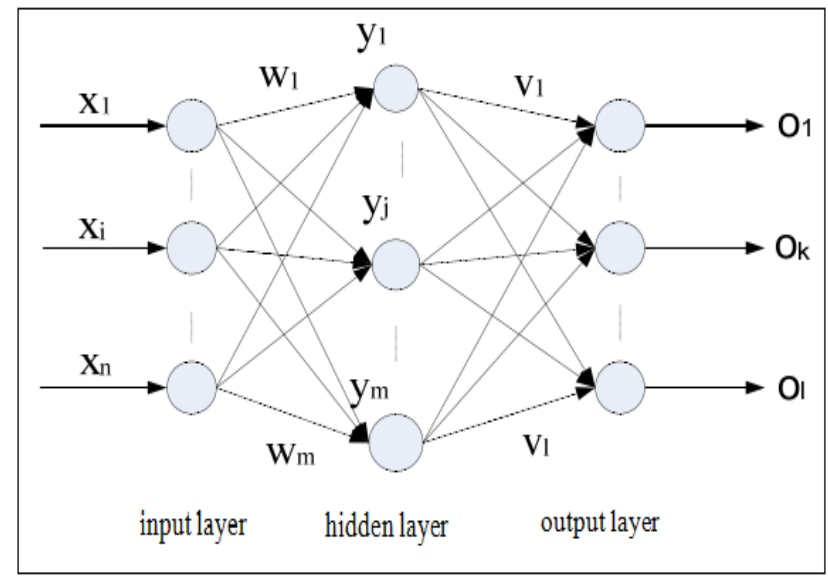

Figure 2. Topology of the BP Neural Network

\section{Particle Swarm Optimization Algorithm}

The particle swarm optimization algorithm is proposed by Kennedy and Eberhart. The algorithm is inspired by the foraging behavior of birds, and is used to solve the optimization problem. In the particle swarm optimization, the solution of each optimization problem is a bird in the search space, and the birds are called particles. Each particle has its own position, velocity, and the fitness value of a function that is determined by the optimized function. The particles follow the current optimal particles in the solution space. In each iteration, the particles update their positions by searching the two extreme values. The first one is the optimal solution, which is found by the particle itself. It is called the individual extreme value point, which is expressed by $p_{\text {best }}$. The other extreme value is the current optimal solution of the whole population. That is the global extreme value, which is expressed by $g_{\text {best }}$. There are $m$ particles that form a group in a D-dimensional search space, and the particle swarm optimization algorithm can be described as follow:

The position of the $i$ th particle is indicated by the $X_{i}=\left(x_{i 1}, x_{i 2}, \cdots, x_{i d}\right),(i=1,2, \cdots, m)$, $V_{i}=\left(v_{i 1}, v_{i 2}, \cdots, v_{i d}\right)$ represents the speed of the $i$ th particle, $p_{b e s t}, i=\left(p_{i 1}, p_{i 2}, \cdots, p_{i d}\right)$ is the optimal location for the $i$ th particle, and $g_{\text {best }}=\left(g_{1}, g_{2}, \cdots, g_{d}\right)$ is the optimal location for all particles in the group. To follow these two optimal values, the particle is updated by the formula (5) and (6) respectively, and the speed and position of the particles are updated to meet the conditions of the end of the iteration.

$$
\begin{gathered}
v_{i d}^{k+1}=\omega^{k} v_{i d}^{k}+c_{1} r_{1}\left(p_{i d}^{k}-x_{i d}^{k}\right)+c_{2} r_{2}\left(g_{d}^{k}-x_{i d}^{k}\right) \\
x_{i d}^{k+1}=x_{i d}^{k}+v_{i d}^{k}
\end{gathered}
$$

\section{The Weight Improved Particle Swarm Neural Network Algorithm}

The variance of the actual output and the expected output of the BP neural network is used as the corresponding error measure:

$$
E=\frac{1}{2} \sum_{k=1}^{n}\left(y_{k}-\hat{y}_{k}\right)^{2}
$$

According to the steepest descent method, we need to adjust the weight $W$ of the hidden layer or the output layer, which can obtain the $E$ 's minimum point: 


$$
\Delta W \propto-\frac{\partial E}{\partial W}
$$

Here, $\frac{\partial E}{\partial W}$ is the growth rate. In order to reduce the error, $\Delta W$ is proportional to and negative growth rate.

Namely.

$$
\Delta W=-L \frac{\partial E}{\partial W}
$$

Among them, $L$ is the ratio coefficient.

Obviously, the function relation of $W$ and $E$ is more direct in the output layer, so the calculation of $\frac{\partial E}{\partial W}$ is relatively simple. But, the function relation of $W$ and $E$ is not a direct relationship in the hidden layer, the calculation of $\frac{\partial E}{\partial W}$ is more complex. So we need to deal with the neurons in the output layer and the hidden layer. Next, we will adjust the $w_{j k}$ of the neurons in the output layer.

$$
\frac{\partial E}{\partial w_{j k}}=\frac{\partial \frac{1}{2} \sum_{k=1}^{n}\left(y_{k}-\hat{y}_{k}\right)^{2}}{\partial w_{j k}}=\left(y_{k}-\hat{y}_{k}\right) \frac{\partial y_{k}}{\partial w_{j k}}
$$

The relationship between $y_{k}$ and $w_{j k}$ is direct in Formula 7, so we just need to obtain the value of $\frac{\partial y_{k}}{\partial w_{j k}}$.

According to the formula 2, we can get

$$
\frac{\partial y_{k}}{\partial w_{j k}}=\frac{\partial f\left(Y_{k}\right)}{\partial w_{j k}}=\frac{\partial\left(\frac{1}{1+e^{-Y_{k}}}\right)}{\partial w_{j k}}=-\left(1+e^{-Y_{k}}\right)^{-2}\left(-e^{-Y_{k}}\right) \frac{\partial Y_{k}}{\partial w_{j k}}=y_{k}\left(1-y_{k}\right) \frac{\partial Y_{k}}{\partial w_{j k}}
$$

And

$$
\frac{\partial Y_{k}}{\partial w_{j k}}=\frac{\partial \sum_{j=1}^{m} h_{j} w_{j k}}{\partial w_{j k}}=\frac{\partial\left(h_{j} w_{j k}\right)}{\partial w_{j k}}=h_{j}
$$

Therefore

$$
\frac{\partial y_{k}}{\partial w_{j k}}=y_{k}\left(1-y_{k}\right) h_{j}
$$

We put the formula 8 into the formula 7, and get the final result of $\frac{\partial E}{\partial w_{j k}}$.

$$
\frac{\partial E}{\partial w_{j k}}=\left(y_{k}-\hat{y}_{k}\right) y_{k}\left(1-y_{k}\right) h_{j}
$$

At this point, the adjustment formula of the $w_{j k}$ of the output layer is:

$$
\Delta w_{j k}=-L_{w_{j k}} \frac{\partial E}{\partial w_{j k}}
$$

In order to reduce the oscillation of the network, we introduce momentum term: 


$$
\Delta w_{j k}(N)=-L_{w_{j k}} \frac{\partial E}{\partial w_{j k}(N)}+\alpha_{w_{j k}} \Delta w_{j k}(N-1)
$$

Here, $N$ is the number of training cycles, $L_{w_{j k}}$ is the learning rate of $w_{j k}$, and $\alpha_{w_{j k}}\left(0 \leq \alpha_{w_{j k}} \leq 1\right)$ is the momentum coefficient..

The momentum term can reduce the sensitivity of the network to the local details of the error surface, and effectively restrain the network fall into the local minimum point. In fact, the momentum term is equivalent to the damping term, which decreases the oscillation tendency of the training and improves the convergence.

Based on the characteristics of particle swarm optimization, the parameters of the neural network are integrated into the PSO model, and the overall optimization is carried out. This can make full use of the global search ability of particle swarm optimization, so that the algorithm of this paper can better play its powerful approximation ability.

\section{Simulation Experiment and Result Analysis}

\subsection{Parameter Definition and Simulation Flow}

The main idea of this paper is based on the historical data which are collected by sensor nodes to predict future data, so as to effectively reduce the amount of data transmission in the network, and save the node energy. The related parameters from the wording of the literature [18], the specific parameters are given in Table 1.

Table 1. The error parameter

\begin{tabular}{|l|l|}
\hline Parameter & instruction \\
\hline$T_{a}$ & The actual value of wireless sensor node detection \\
\hline$T_{f}$ & The predictive value of the algorithm \\
\hline$\varepsilon$ & Prediction error \\
\hline$C$ & Error threshold \\
\hline$\varepsilon \leq C$ & Prediction success, using the predictive value for transmission \\
\hline$\varepsilon>C$ & Prediction without success, using the detection value for transmission \\
\hline
\end{tabular}

In the architecture of wireless sensor networks, the working process of the nodes in the network is divided into three stages: the historical data collection, the future data prediction, and the predicted results test. At the initial stage of the system operation, because there is not enough historical data, the nodes cannot predict. At this time, each node of the wireless sensor is in the stage of historical data collection. After collecting enough historical data, the node enters into the future data prediction stage. According to the historical data and the forecast model, the nodes calculate the predicted value of the next collected data. By comparing the prediction error and the error threshold, the node decides whether to update the model and data transmission.

\subsection{Training Model}

The effectiveness of the data fusion technique in this paper depends on the accuracy of the prediction algorithm. The purpose of validation is to test the accuracy of the three prediction algorithms, and to analyze and compare them. Five meteorological factors are selected in this study. They are temperature $(\mathrm{T})$, relative humidity $(\mathrm{RH})$, air pressure $(\mathrm{P})$, wind speed $(\mathrm{F})$, precipitation $(\mathrm{R})$. We set up a sample set of daily average value of five meteorological factors, and use them as the input value in the training of BP neural network. The output value is PM2.5 concentration. Each node has 365 sets of data in 2015. Then, we define the first 360 sets of indicators as training samples, and the final 5 groups of indicators is defined as the test sample. 
In order to make the index have comparability, and to speed up the convergence rate of the neural network, this paper has carried on the normalized processing to each index:

1) For qualitative indicators: using expert scoring method to determine its data, and we have a normalized treatment of various indicators.

2) For quantitative indicators: the following formula is used to normalize.

$$
x_{i}=\frac{x_{i}-x_{i \min }}{x_{i \max }-x_{\min }}
$$

Where, the normalized values for the $i$ th indicator is $x_{i}$, the minimum value of the $i$ th indicator is $x_{i \min }$, and the maximum value of the $i$ th indicator is $x_{i \max }$.

Next, we train the sample set that has been orthogonal to find thenumber of hidden layer nodes when the fitting error get the minimum value, so as to complete the construction of the improved PSO neural network model.

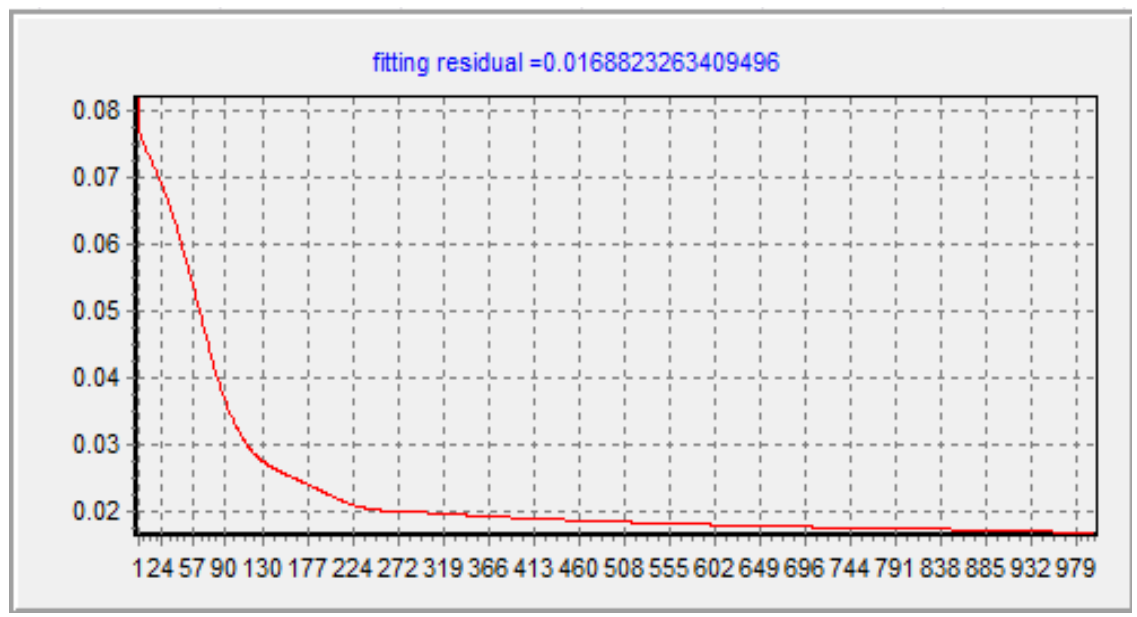

Figure 3. The Number of Hidden Layer Node is $\mathbf{3}$ in Neural Network Training

As can be seen from Figure 3, when number of hidden layer node of the BP network is 3 , the mean square error reaches the minimum, which is 0.0169 . Thus, an improved BP neural network model trained with 3 hidden layer nodes is completed.

\subsection{Prediction and Performance Analysis}

First of all, we give the monitoring data of PM2.5 in 2015 in Figure 4. As can be seen from the figure, the PM2.5 value in the city of Beijing is higher in spring and winter, but lower in summer and autumn. This shows that meteorological conditions are the main factors that affect the seasonal variation of PM2.5. 


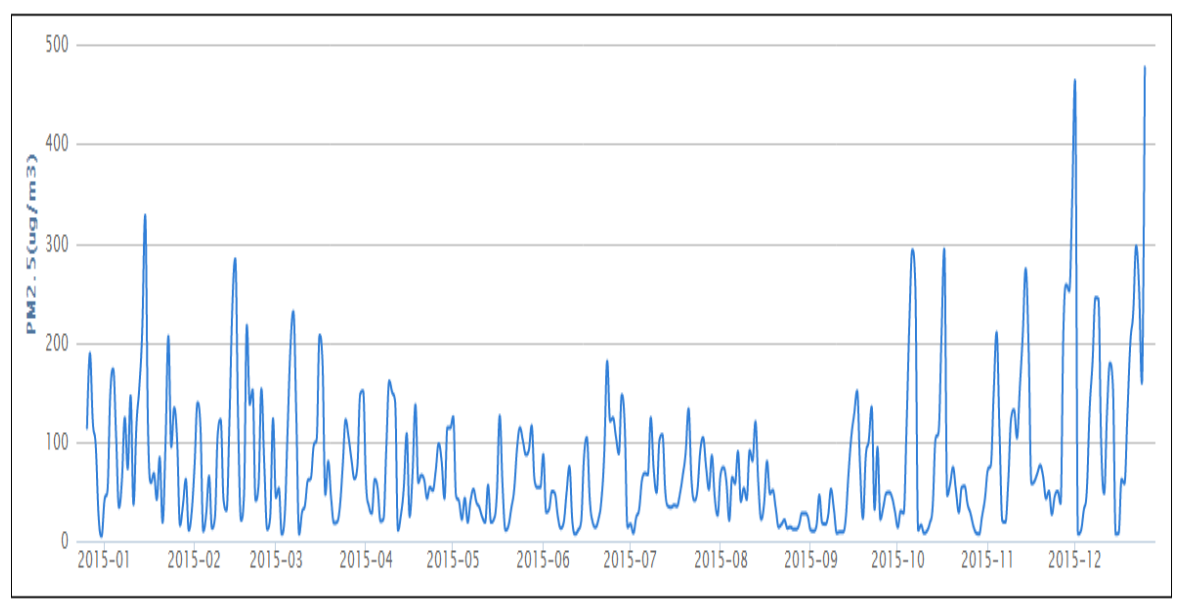

Figure 4. PM2.5 Monitoring Data in Beijing in 2015

Secondly, due to the limited length of this paper, we only use the relationship between temperature and PM2.5 for example. From Figure 5 we can see that the relationship is closely related between annual temperature and the PM2.5. In spring and winter, when the temperature is low, the PM2.5 is more than 300. And when the temperature is higher, the PM2.5 less than 100 is very frequent. At this time the air quality is excellent. At this time, the air quality is excellent. The rest of the four factors also has a very close relationship with PM2.5. Therefore, this paper selects these five factors as the sample data set, and then put them into the neural network prediction model which is established in this paper.

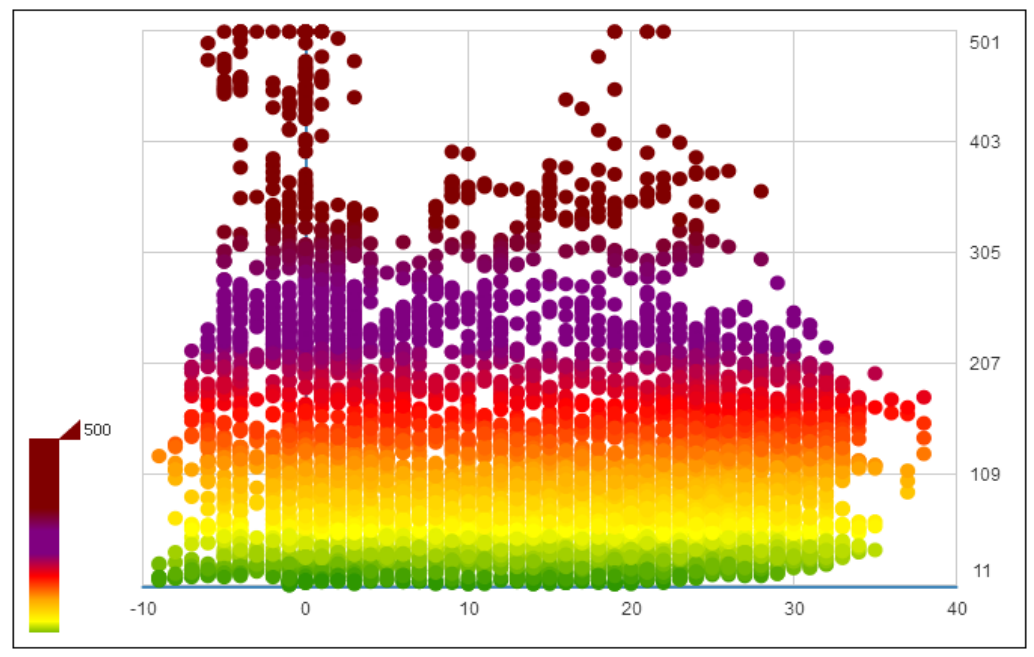

Figure 5. Relationship between Temperature and PM2.5

Thirdly, we use three different methods to predict the value of PM2.5. After that, we consider the success degree of each prediction model. Then, the algorithm of this paper, multiple regression algorithm and grey prediction method are analyzed and compared under different error thresholds. Figure 6-8 shows that the error threshold is increased from $1 \mu \mathrm{g} / \mathrm{m}^{3}$ to $5 \mu \mathrm{g} / \mathrm{m}^{3}$, and the prediction success degree of the three algorithms are changed. 


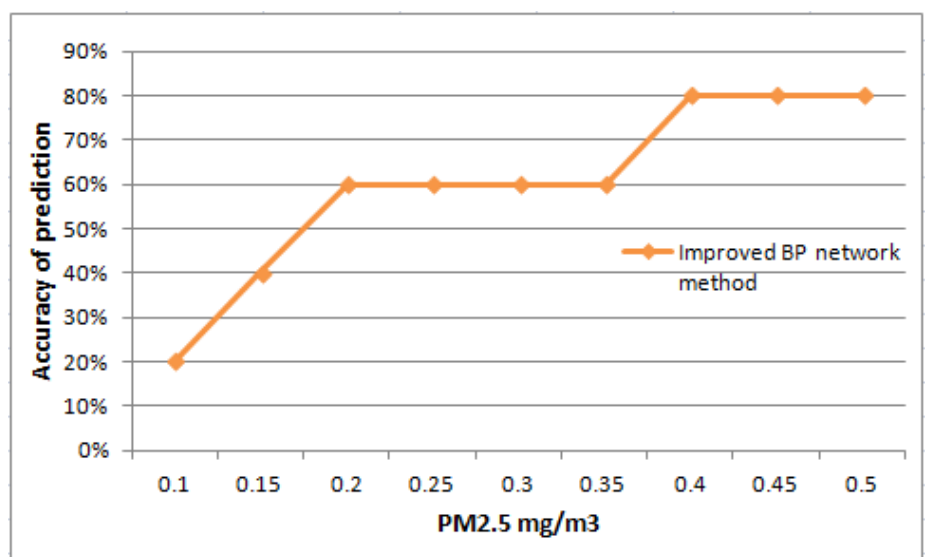

Figure 6. Success Degree of Prediction of the Improved BP Network

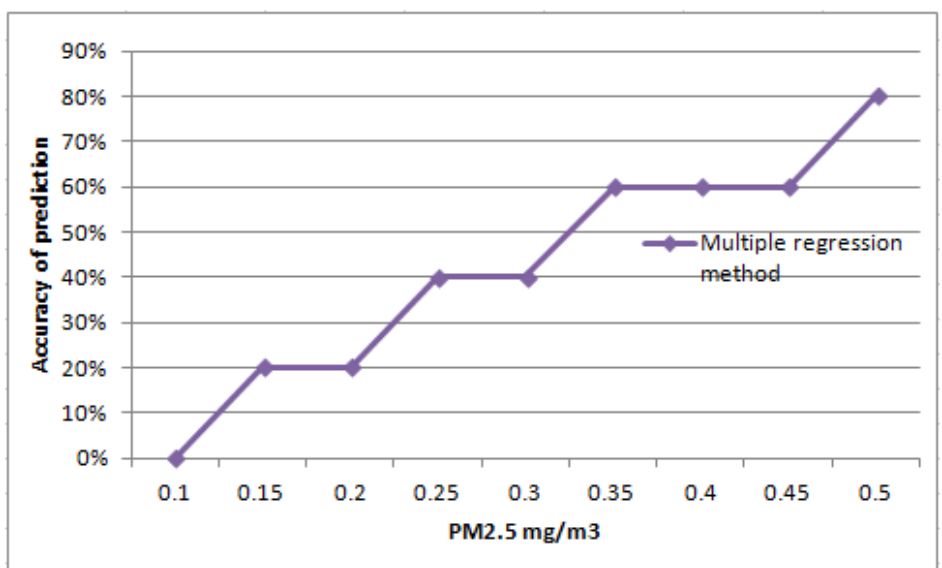

Figure 7. Success Degree of Prediction of the Multiple Regression

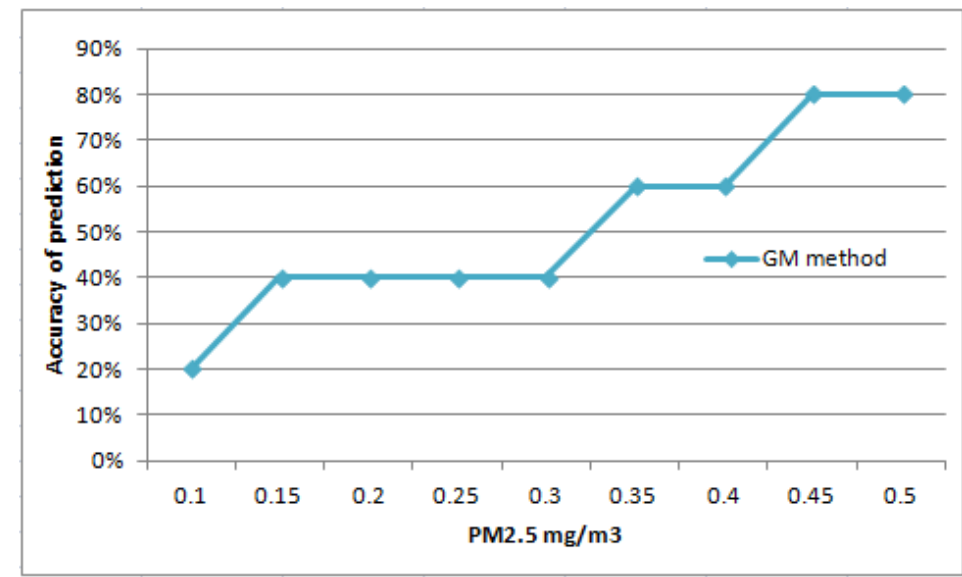

Figure 8. Success Degree of Prediction of the GM Model

As can be seen from the figure 6-8, with the increase of the error threshold, the success degree of the each prediction algorithm shows a rising trend. In the three algorithms, the improved PSO neural network prediction algorithm is superior to other algorithms. With the increase of the error threshold, the success degree of the gray prediction algorithm is close to this article' algorithm. When the error threshold is $1 \mu \mathrm{g} / \mathrm{m}^{3}$, the prediction 
success degree of the improved PSO neural network model is $20 \%$, the prediction success degree of the multiple regression algorithm is 0 , and the prediction success degree of the grey prediction algorithm is $20 \%$. When the error threshold is $5 \mu \mathrm{g} / \mathrm{m}^{3}$, the prediction success degree of the three models is $80 \%$. In the range of $2 \mu \mathrm{g} / \mathrm{m}^{3}-3 \mu \mathrm{g} / \mathrm{m}^{3}$, the prediction accuracy of the three algorithms has a significant improvement.

Finally, in order to quantitatively analyze the energy saving benefits brought by the data fusion technology which is based on prediction, we realize the algorithm of this article, multiple regression algorithm and grey prediction method in the network layer of wireless sensor architecture. Under different error thresholds, we analyze and compare the energy consumption of the nodes when we use the three prediction algorithms and not to use the prediction algorithm. Figure 9 shows the relationship between the average energy consumption and the error threshold of the wireless sensor nodes.

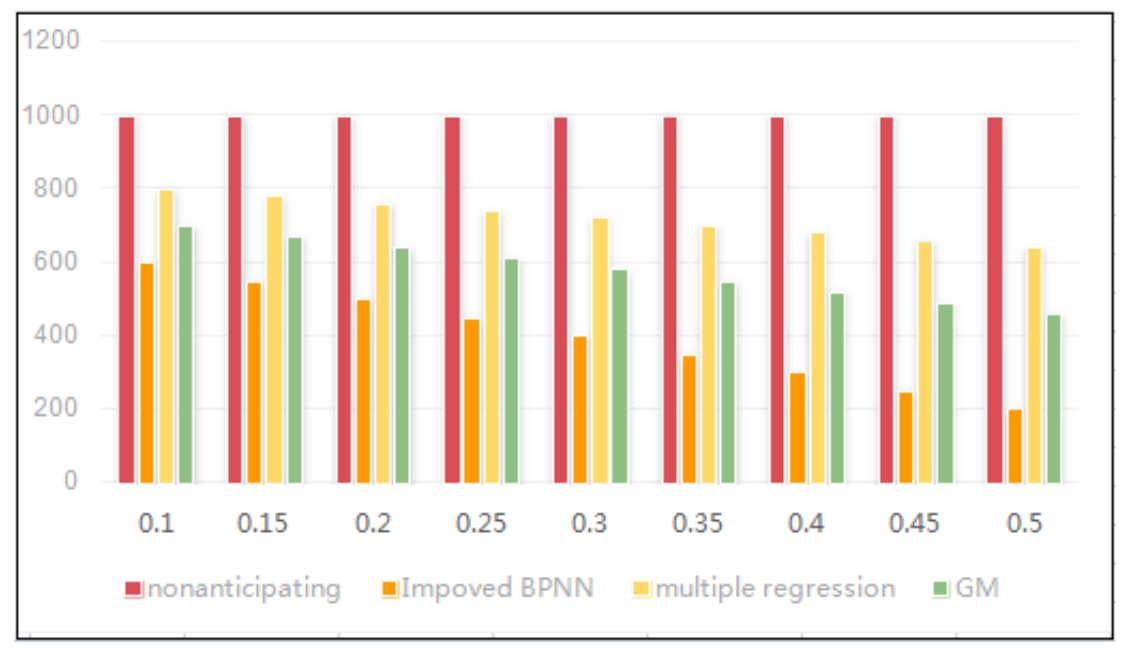

Figure 9. Comparison Diagram of Energy Consumption

As can be seen from Figure 9, in the interval of $1 \mu \mathrm{g} / \mathrm{m}^{3}-5 \mu \mathrm{g} / \mathrm{m}^{3}$, the prediction algorithm based on the improved particle swarm optimization neural network can achieve the purpose of saving energy. When the error threshold is greater than or equal to $3 \mu \mathrm{g} / \mathrm{m}^{3}$, the other two algorithms can achieve the purpose of saving energy. Therefore, the method can be used to save energy in wireless sensor data transmission.

\section{Conclusion}

In this paper, a data prediction model of wireless sensor network based on weight improved particle swarm optimization neural network algorithm is proposed. In view of the deficiency of the traditional BP neural network model, this paper combines with the characteristics of the data prediction model, and the BP neural network model is improved and integrated. After that, we train the neural network's sample set, and add the momentum item to correct the weight, so that the neural network can be predicted more quickly and accurately. The main idea of this paper is to predict the future data based on the historical data which are collected by sensor nodes, so as to achieve the purpose of reducing the amount of data transmission in the network and saving the energy of nodes. Finally, the experimental results show that the improved particle swarm optimization algorithm based on weight improved particle swarm optimization neural network algorithm has higher accuracy than the multiple regression method and the grey prediction method. In addition, the method can be used to effectively save energy in wireless sensor data transmission. 


\section{Acknowledgement}

This study was supported by the projects of Science Technology Department of Zhejiang Province (No.2015C33230) and Quzhou University teachers team construction fund (No. XNZQ201311) and Open laboratory project of Quzhou University (No. KFXM201511) and Quzhou Science and Technology Bureau (No. 2014192).

\section{Reference}

[1] I.F. Akyildiz, W. Su and Y. Sankara Subramaniam, "A Survey on Sensor Networks [J]", IEEE Communications Magazine, vol. 40, no. 8, (2002), pp. 102-114.

[2] G. Werner-Allen, K. Lorincz and M. Welsh, "Deploying a wireless sensor network on an active volcano [J]", IEEE Internet Computing, vol. 10, no. 22, (2006), pp. 18-25.

[3] F. Viani, P. Rocca and G. Oliveri, "Localization, tracking, and imaging of targets in wireless sensor networks: an invited review [J]", Radio Science, (2011).

[4] E.E. Emeka and O.F. Abraham, "A survey of system architecture requirements for health care-based wireless sensor networks [J]", Sensors, vol. 11, no. 5, (2011), pp. 4875-4898.

[5] L. Fernando, G. Antonio-Javier and G. Felipe, "A comprehensive approach to WSN-based ITS applications: a survey [J]", Sensors, vol. 11, no. 11, (2011), pp. 10220-10265.

[6] A. Cristina, S. Pedro and I. Andrés, "Wireless sensor networks for oceanographic monitoring: a systematic review [J]", Sensors, vol. 10, no. 7, (2010), pp. 6948-6968.

[7] C. Intanagonwiwat, R. Govindan and D. Estrin, "Directed diffusion: a scalable and robust communication paradigm for sensor networks [C]", ACM/IEEE International Conference on Mobile Computing and Networks, (2000).

[8] J. Heidemann, F. Silva and C. Intanagonwiwat, "Building efficient wireless sensor networks with lowlevel naming [C]", 18th ACM Symposium on Operating Systems Principles, (2001).

[9] B. Krishnamachari, D. Estrin and S. Wicker, "Modelling data- centric routing in wireless sensor networks [C]", In Proceeding of IEEE Infocom, (2002).

[10] G. Anastasi, M. Conti and M.D. Francesco, "Energy conservation in wireless sensor networks [J]", Ad Hoc Networks, vol. 7, no. 3, (2009), pp. 537- 568.

[11] X. Wang, J. Ma and S. Wang, "Time series forecasting for energy-efficient organization of wireless sensor networks [J]", Sensors, vol. 7, no. 9, (2007), pp. 1766-1792.

[12] P.M.T. Broersen, "Automatic identification of time-series models from long autoregressive models [J]", IEEE Transactions on Instrumentation and Measurement, vol. 54, no. 5, (2005), pp. 1862-1868.

[13] J. Zuo, C. Tang and T. Zhang, "Mining Predicate Association Rule by Gene Expression Programming [C]", WAIM02, LNCS, Berlin: Springer-Verlag, (2002), pp. 92 -103.

[14] X. Wang, Z.-H. Qian and Z.-C. Hu, "Research on RFID anti-collision algorithms based on binary tree [J]", Journal on Communications, vol. 31, no. 6, (2010), pp. 49-57.

[15] P.-K. Liao, M.-K. Chang and C.J. Kuo, "A statistical approach to contour line estimation in wireless sensor networks with practical considerations [J]", IEEE Transactions on Vehicular Technology, vol. 58, no. 7, (2009), pp. 3579-3595.

[16] L.M. Sun, J.Z. Li and Y. Chen, "Wireless Sensor Networks [M]", Beijing: Tsinghua University Press, (2005), pp. 4-10.

[17] I.F. Akyildiz, M. Tommaso and R. Kaushik, "Wireless multimedia sensor networks: a survey [J]", IEEE Wireless Communications, vol. 14, no. 6, (2007), pp. 32-39.

[18] C. Hui and L. Cui, "Forecast based temporal data aggregation in wireless sensor networks [J]", Computer Engineering and Applications, vol. 43, no. 21, (2007), pp. 121-125. 
International Journal of Future Generation Communication and Networking Vol. 9, No. 3 (2016) 